\title{
Open Science
}

Accelerate Open Science project

\section{Source}

Accelerate Open Science project. What is Open Science?

'Open Science' stands for the transition to a new, more open and participatory way of conducting, publishing and evaluating scholarly research. Central to this concept is the goal of increasing cooperation and transparency in all research stages. This is achieved, among other ways, by sharing research data, publications, tools and results as early and open as possible.

Open Science leads to more robust scientific results, to more efficient research and (faster) access to scientific results for everyone. This results in turn in greater societal and economic impact. 